\title{
Clinical types of checkpoint inhibitor-related pneumonitis in lung cancer patients: a multicenter experience
}

\author{
Xinqing Lin ${ }^{1,2 \#}$, Haiyi Deng ${ }^{1 \#}$, Likun Chen ${ }^{3,4,5 \#}$, Di Wu ${ }^{6}$, Xiaobo Chen ${ }^{1}$, Yilin Yang ${ }^{1}$, Tao Chen ${ }^{1}$, \\ Xiaohong Xie ${ }^{1}$, Zhanhong Xie ${ }^{1}$, Ming Liu ${ }^{1}$, Ming Ouyang ${ }^{1}$, Yinyin Qin ${ }^{1}$, Shiyue $\mathrm{Li}^{1}$, Nanshan Zhong ${ }^{1}$, \\ Jeffrey P. Gregg ${ }^{7}$, Nobuyuki Horita ${ }^{8}$, Yong Song ${ }^{2,9}$, Chengzhi Zhou ${ }^{1}$
}

${ }^{1}$ State Key Laboratory of Respiratory Disease, National Clinical Research Centre for Respiratory Disease, Guangzhou Institute of Respiratory Health, First Affiliated Hospital, Guangzhou Medical University, Guangzhou, China; ${ }^{2}$ The First School of Clinical Medicine, Southern Medical University, Guangzhou, China; ${ }^{3}$ Department of Medical Oncology, Sun Yat-Sen University Cancer Center, Guangzhou, China; ${ }^{4}$ State Key Laboratory of Oncology in South China, Guangzhou, China; ${ }^{5}$ Collaborative Innovation Center for Cancer Medicine, Guangzhou, China; ${ }^{6}$ Shenzhen People's Hospital, The Second Clinical Medical College of Jinan University, The First Affiliated Hospital, Southern University of Science and Technology, Shenzhen, Guangdong, China; ${ }^{7}$ Department of Pathology and Laboratory Medicine, University of California, Davis Medical Center, Sacramento, CA, USA; ${ }^{8}$ Department of Pulmonology, Yokohama City University Graduate School of Medicine, Yokohama, Japan; ${ }^{9}$ Department of Respiratory and Critical Care Medicine, Jinling Hospital, Nanjing, China

Contributions: (I) Conception and design: X Lin, H Deng, Y Song, C Zhou, N Zhong; (II) Administrative support: Guangdong High-Level University Clinical Cultivation Project, Guangzhou Science and Technology Program key projects, and Wu Jieping Medical Foundation; (III) Provision of study materials or patients: X Lin, L Chen, D Wu, X Xie, Y Song, Y Qin, S Li, X Chen, Z Xie, M Ouyang, C Zhou; (IV) Collection and assembly of data: H Deng, M Liu, Y Yang, T Chen, L Chen, D Wu; (V) Data analysis and interpretation: All authors; (VI) Manuscript writing: All authors; (VII) Final approval of manuscript: All authors.

\#These authors contributed equally to this work.

Correspondence to: Chengzhi Zhou. Guangzhou Institute of Respiratory Health, State Key Laboratory of Respiratory Disease, First Affiliated Hospital, Guangzhou Medical University, 151 Yanjiang Road, Guangzhou 510120, China. Email: doctorzcz@163.com; Yong Song. Department of Respiratory and Critical Care Medicine, Jinling Hospital, The First School of Clinical Medicine, Southern Medical University, 305 East Zhongshan Road, Nanjing 210002, China. Email: yong.song@nju.edu.cn.

Background: Checkpoint inhibitor-related pneumonitis (CIP) is not well classified according to clinical factors. We propose different clinical sub-types of CIP based on clinical factors and investigated the corresponding clinical features, treatments, and outcomes.

Methods: We conducted a multicenter retrospective study of patients with lung cancer (including nonsmall cell lung cancer and small cell lung cancer) who developed CIP. The clinical characteristics, radiologic features, treatments, and outcomes of CIP were analyzed.

Results: A total of 55 patients developed CIP and were classified into 3 groups as follows: 21 in the pure type (PT) group, 14 in the induced type (IT) group, and 20 in the mixed type (MT) group. The incidence of severe (grade 3-5) pneumonitis was significantly higher in the IT group than in the PT and MT groups (71.4\% vs. $14.3 \%$ vs. $50.0 \%, \mathrm{P}=0.002$ ). Antiviral therapy was significantly more frequent in the IT group than in the PT and MT groups. Antibiotic therapy was administered in $23.8 \%, 71.4 \%$, and $80.0 \%$ of patients with the PT, IT, and MT, respectively. The improvement time in the PT group was longer than that in the IT and MT groups ( 0.9 vs. 0.5 vs. 0.3 months, $\mathrm{P}=0.028)$. Patients with the $\mathrm{PT}$ had a better tumor response to immune checkpoint inhibitors (ICIs) than those with the other 2 types [overall response rate (ORR), $78 \% v s$. $31 \%$ vs. $44 \%, \mathrm{P}=0.027]$.

Conclusions: The clinical classification of CIP may favor strategies for treatments and predict the tumor response to ICIs.

Keywords: Immune checkpoint inhibitor (ICI); immune-related adverse events; checkpoint inhibitor-related pneumonitis (CIP); lung cancer, clinical types 
Submitted Sep 24, 2020. Accepted for publication Jan 02, 2021.

doi: $10.21037 /$ tlcr-20-1258

View this article at: http://dx.doi.org/10.21037/tlcr-20-1258

\section{Introduction}

In the past decade, the overall survival (OS) rate of patients with lung cancer has improved significantly as a result of immune checkpoint inhibitors (ICIs) $(1,2)$. However, ICIs are also associated with immune-related adverse events (irAEs) and even fatal adverse events (FAEs) (3). The overall incidence of FAEs in patients treated with programmed death-1/programmed death-ligand 1 (PD$1 / \mathrm{PD}-\mathrm{L} 1)$ inhibitors was $0.43 \%$, mainly involving the respiratory system (46.2\%) (4). The clinical manifestations of checkpoint inhibitor-related pneumonitis (CIP) vary, ranging from occult respiratory symptoms with subacute or subclinical asymptomatic disease to rapid acute respiratory failure, even resulting in death (5-7). The patterns of the radiologic manifestations of CIP aslo vary and include scattered or diffuse ground-glass opacity (GGO), consolidation, interlobular septal thickening, reticular shadow, extensive branch expansion, nodules, and fiber strip shadow (8). The CIP is usually graded according to the symptoms and/or imaging manifestations and Common Terminology Criteria for Adverse Events (CTCAE; version 4.0) (9-11). In clinical practice, it has been found that in the same grade of CIP, there may be both dramatic exacerbation and rapid improvement. There are also great differences in the improvement time of CIP.

Previous studies have shown that OS and progressionfree survival (PFS) is significantly longer in patients with irAEs than in those without irAEs. However, in subgroup analysis, CIP was not significantly associated with the efficacy of immunotherapy $(12,13)$. Conversely, a study showed that grade 1-2 CIP was associated with increased ICI efficacy, yet severe grade CIP was not (14). A metaanalysis showed that CIP is significantly heterogeneous $\left(\mathrm{I}^{2}=70.0 \%, \mathrm{P}<0.001\right)$ in OS analysis but not in other organs (15).

One of the reasons for the heterogeneity of CIP may due to the structure of the lung. The lungs communicate with the outside world and have 2 blood supply systems connected with the blood and lymph circulation of other organs, so they are continuously contending with factors such as microbes. Studies have reported that infections in ICI-treated patients occur mainly in the lungs $(16,17)$. A meta-analysis showed that lung cancer patients treated with
ICIs were at risk not only for CIP, but also for infectious pneumonia (18).

Studies have shown that immunotherapy combined with radiotherapy increases the incidence of CIP $(19,20)$. Case reports have described the development of severe CIP in patients receiving combination therapy with thoracic radiotherapy and ICI (21). Similarly, in one case report, a patient with lung cancer developed severe pneumonitis, complicated with bacterial pneumonia and radiation-related pneumonitis (22).

We hypothesize that the heterogeneity of pneumonitis may be associated with 2 hypotheses; antitumor responsedependent mechanisms, and response-independent mechanisms (23). Studies have shown that CIP may be triggered by antigens common to tumors and inflammatory organs (24,25). In addition, autoreactive T cells, autoantibodies, and cytokines produced by the antitumor response act on inflammatory organs (26). Contrastingly, the microbiome (virus, bacteria) may cause the original specific antigen to be exposed. The mechanisms are also different due to microbial diversity and composition $(27,28)$. Our previous study showed that cytomegalovirus (CMV) reactivation was associated with CIP (29). In a patient with fatal ICI-induced encephalitis, Epstein-Barr virus (EBV)specific T-cell receptors and EBV-positive lymphocytes were identified in the cortex and meninges, suggesting that EBV was associated with irAEs (30). However, the prevailing guidelines do not target microbial therapy.

To make the treatment strategies of CIP more individualized and effective, we hypothesize that the diversity of CIP can be categorized into distinct types according to clinical circumstances. Thus, we analyzed the clinical characteristics, managements, and outcomes of the different types of CIP and retrospectively evaluated the feasibility of defining various types.

We present the following article in accordance with the STROBE reporting checklist (available at http://dx.doi. org/10.21037/tlcr-20-1258).

\section{Methods}

\section{Patients}

This multicenter, retrospective, observational study 
was conducted in 3 centers [First Affiliated Hospital of Guangzhou Medical University (FHGMU), Collaborative Innovation Center for Cancer Medicine (CICM), and Shenzhen People's Hospital (SPH)] in the southern region of China. All patients were diagnosed with primary lung cancer [according to the 2015 World Health Organization Classification of Lung Tumors (31)] and received $\geq 1$ dose of ICI between February 2018 and August 2020. The diagnosis of CIP was based on typical clinical features, physiological and chest computerized tomography (CT) scan findings $(32,33)$. All procedures performed in this study involving human participants were in accordance with the Declaration of Helsinki (as revised in 2013). Institutional review board/ethics committee approval was obtained from the Institutional Review Board of the First Affiliated Hospital of Guangzhou Medical University (Guangzhou, Guangdong, China) (No. 2020-95). Individual consent for this retrospective analysis was waived.

\section{Data collection and study assessment}

For all participants, the following data were collected retrospectively: patient demographics, time course of CIP, Eastern Cooperative Oncology Group Performance Status (ECOG PS), modified Medical Research Council Dyspnea Scale (mMRC), maximum CIP grade, imaging features, laboratory findings, and treatments and outcomes of CIP. The clinical data of each patient were recorded and verified by trained professionals.

We classified CIP into 5 subtypes in terms of imaging lesions: cryptogenic organizing pneumonitis (COP), Ground-glass opacification/opacity (GGO), interstitial, hypersensitivity, and pneumonitis not otherwise specified (NOS) according to previous reports (34). According to the American Thoracic Society (ATS) and the European Respiratory Society (ERS) classification of interstitial pneumonia, radiographic patterns of pneumonitis were also classified as usual interstitial pneumonia (UIP), nonspecific interstitial pneumonia (NSIP), the cryptogenic organizing pneumonia (COP) pattern, acute interstitial pneumonia (AIP)/acute respiratory distress syndrome (ARDS), and hypersensitivity pneumonitis (HP) $(35,36)$. Results of procalcitonin (PCT), routine hematological, chemistry, and microbiology (including CMV, EBV, Mycobacterium tuberculosis, bacteria, and fungi) related tests were documented at the onset of CIP.

The severity of CIP was graded according to the Common Toxicity Criteria for Adverse Events (CTCAE version 4.0). The ECOG PS and mMRC scores were evaluated at the most severe pneumonitis. Improvement of pneumonitis was defined as the improvement of symptoms, reduction of oxygen requirement, or improvement of radiographic infiltrates. Inversely, worsening was defined as the exacerbation of symptoms, increased oxygen requirement, or increased radiographic infiltrates. Tumor response was assessed according to the Response Evaluation Criteria in Solid Tumors (RECIST version 1.1) (37). The complete overall response rate (ORR) was defined as the percentage of participants achieving complete response (CR) and partial response $(\mathrm{PR})$.

\section{Statistical analysis}

Continuous and categorical data are summarized as medians (ranges) and frequencies (percentages), respectively. Analysis of variance or the Kruskal-Wallis H test was used to analyze continuous variables. The categorical variables were compared with chi-squared $\left(\chi^{2}\right)$ or Fisher's exact tests. Spearman's rank correlation was used for correlation analysis of 2 ordered categorical variables. The contingency coefficient was calculated to analyze the correlation between AIP-ARDS and the grade of pneumonitis. Logistic regression was used to identify factors associated with the improvement rate of CIP by univariate and multivariable analyses. We conducted statistical analyses using SPSS version 25 (IBM Software, Armonk, NY, USA). A P value $<0.05$ was considered statistically significant.

\section{Results}

\section{Participants}

A total of 55 patients who developed CIP following ICIs treatment were included at FHGMU $(n=49)$, CICM $(n=5)$, and SPH $(n=1)$. The patient demographics are shown in Table 1. At the time of initiation of CIP, the median age was 62 [18-85] years; most participants were male (80.0\%), 28 were smokers, 8 had pre-existing lung diseases (emphysema, chronic obstructive pulmonary disease, and pulmonary fibrosis), and 12 participants had received radiotherapy before CIP.

All 55 patients had primary lung cancer $(45.5 \%$ squamous-cell carcinoma, $23.6 \%$ adenocarcinoma, $14.5 \%$ small cell carcinoma, $16.4 \%$ others). Anti-PD-L1 treatment had been received by 1 participant, and 54 had received anti-PD1 treatment $(98.2 \%) ; 37$ (67.3\%) received ICIs 
Table 1 Characteristics of the patients at baseline

\begin{tabular}{lc}
\hline Characteristics & No. of patients (\%) \\
\hline Age, y; median [range] & $62[18-85]$ \\
Gender (male/female) & $44 / 11$ \\
Smoking status & \\
Current/former & $28(50.9)$ \\
Never & $27(49.1)$ \\
Pre-existing lung diseases & $8(14.5)$ \\
History of radiotherapy & $12(21.8)$ \\
Treatment with ICI & \\
Monotherapy & $18(32.7)$ \\
Combined therapy & $37(67.3)$ \\
Histology & \\
Squamous & $15(27.3)$ \\
Adenocarcinomas & $7(12.7)$ \\
Small cell carcinoma & $25(45.5)$ \\
Others & $13(23.6)$ \\
Tumor staging & $8(14.5)$ \\
III & $9(16.4)$ \\
Ist line & $2(3.6)$ \\
\hline
\end{tabular}

$\mathrm{ICl}$, immune checkpoint inhibitor.

combination therapy, and $18(32.7 \%)$ were treated with ICI monotherapy. Most patients (92.7\%) had stage III/ IV disease when immunotherapy was introduced, and 2 patients with stage II disease received immunotherapy as neoadjuvant therapy.

\section{Definition of the clinical CIP types}

According to the clinical factors, we divided 55 patients into 3 types: pure type (PT), induced type (IT), and mixed type (MT) pneumonitis (Figure 1). The PT was defined

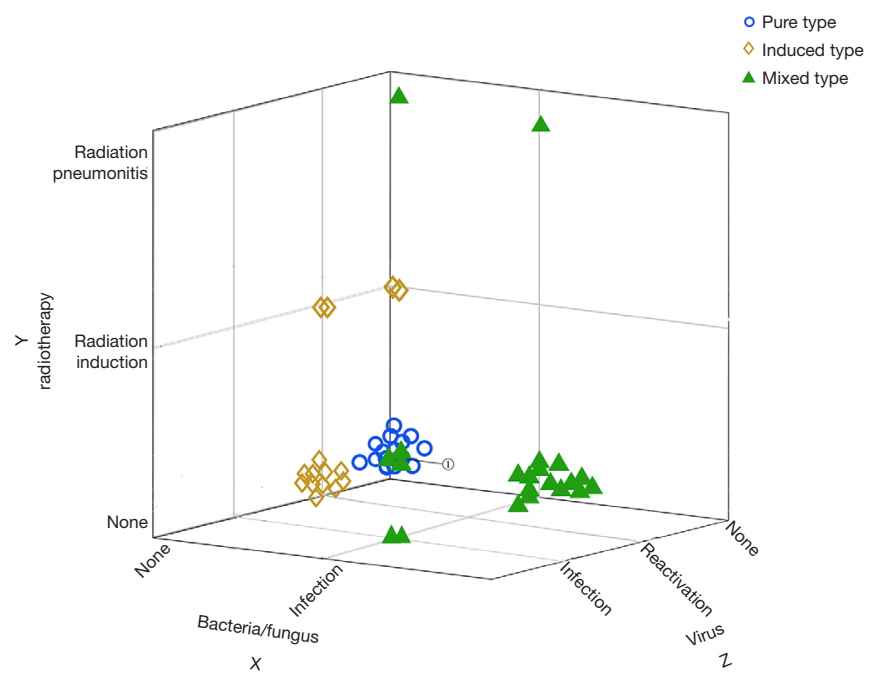

Figure 1 Grouping type based on clinical factors. The $\mathrm{X}$-axis (bacterial or fungal infection). The Y-axis shows the relationship between radiotherapy and pneumonitis. The $\mathrm{Z}$-axis represents virus infection or reactivation. ${ }^{(1)}$, the 3 patients developed pneumonitis with disease progression and were classified as mixed type.

as idiopathic, with or without autoimmune disease (AID). The IT was defined as having distinct etiologies, such as radiotherapy, CMV, or EBV reactivation, and producing specific antigens, which lead to specific immune cell activation and then to CIP, without evidence of organ damage caused by virus or radiotherapy. The MT was defined as CIP combined with infectious pneumonia (bacteria, fungus, or other organisms), tumor progression (including pseudoprogression or hyperprogression), or radiation-related pneumonitis.

\section{Clinical and radiological features of CIP}

The severity of CIP was grade 1 in 10 participants, grade 2 in 22 participants, grade 3 in 12 participants, grade 4 in 8 participants, and there were 3 fatalaties (grade 5). The median time from the onset of immunotherapy to the development of CIP was 2.6 months (0.2-15.8 months). The main clinical manifestations of pneumonitis were cough, expectoration, and shortness of breath.

Of the 55 participants, 21 had the PT (38.2\%), 14 had the IT (25.4\%), and 20 had the MT (36.4\%). Table 2 summarizes the clinical characteristics of the 3 types of CIP. The proportion of fever in the MT was significantly 
Table 2 Clinical characteristics of the new types

\begin{tabular}{|c|c|c|c|c|}
\hline Characteristic & Pure type $(n=21)$ & Induced type $(n=14)$ & Mixed type $(n=20)$ & $P$ value \\
\hline \multicolumn{5}{|l|}{ Symptoms } \\
\hline Fever & $1(4.8)$ & $4(28.6)$ & $13(65.0)$ & $<0.001$ \\
\hline Cough & $14(66.7)$ & $12(85.7)$ & $18(90.0)$ & 0.068 \\
\hline Shortness of breath & $6(28.6)$ & 9 (64.3) & $16(80.0)$ & 0.001 \\
\hline ECOG PS & & & & 0.002 \\
\hline $0-1$ & $14(66.7)$ & $2(14.3)$ & $4(20.0)$ & \\
\hline $2-4$ & 7 (33.3) & $10(71.4)$ & $15(75.0)$ & \\
\hline $0-1$ & $13(61.9)$ & $2(14.3)$ & $2(10.0)$ & \\
\hline $2-4$ & $8(38.1)$ & $12(85.7)$ & $18(90.0)$ & \\
\hline Grade & & & & 0.020 \\
\hline 1 & $8(38.1)$ & $1(7.1)$ & $1(5.0)$ & \\
\hline 2 & $10(47.6)$ & $3(21.4)$ & $9(45.0)$ & \\
\hline 3 & $1(4.8)$ & $5(35.7)$ & $6(30.0)$ & \\
\hline 4 & $2(9.5)$ & $3(21.4)$ & $3(15.0)$ & \\
\hline 5 & 0 & $2(14.3)$ & $1(5.0)$ & \\
\hline COP & 7 (33.3) & $1(7.1)$ & $2(10.0)$ & \\
\hline GGO & $6(28.6)$ & $11(78.6)$ & $7(35.0)$ & \\
\hline NSIP & $4(19.0)$ & $2(14.3)$ & $8(40.0)$ & \\
\hline NOS & $4(19.0)$ & 0 & $3(15.0)$ & \\
\hline
\end{tabular}

ECOG PS, Eastern Cooperative Oncology Group performance status; mMRC, modified Medical Research Council Dyspnea Scale; PCT, procalcitonin; WBC, white blood cell count; pp65, phosphoprotein 65; COP, cryptogenic organizing pneumonitis; GGO, ground glass opacities; NSIP, non-specific interstitial pneumonia; NOS, pneumonitis not otherwise specified.

higher than in the PT and IT groups $(65.0 \%, 4.8 \%$, and $28.6 \%$, respectively, $\mathrm{P}<0.001)$. Grade $3-5$ CIP occurred in 3 participants (14.3\%) with the PT, $10(71.4 \%)$ with the IT, and 10 (50.0\%) with the MT (Figure 2). The ECOG PS at the most severe pneumonitis was significantly different among the 3 groups $(\mathrm{P}=0.002)$, and the $\mathrm{PS}$ in the PT group was mainly $0-1(66.7 \%)$, while those in the IT group (71.4\%) and MT group (75.0\%) were mostly $2-4$. The incidence of $\mathrm{mMRC}$ scores of 2-4 was lower in the PT group than in the IT and MT groups $(\mathrm{P}=0.001)$. 
Procalcitonin (PCT) and white blood cells count (WBC) were higher in the MT group than in the PT and IT groups.

A strong positive association was found between the PS score and the grade of CIP [determined by Spearman's rank correlation coefficient $\left.\left(r_{s}\right)=0.907, P<0.001\right]$. Similarly, the $\mathrm{mMRC}$ score was higher in patients with a higher grade of CIP $\left(r_{s}=0.873, \mathrm{P}<0.001\right)$.

The most predominant lesion found on chest CT was GGO lesion (43.6\%), followed by NSIP (25.5\%), COP $(18.2 \%)$, and NOS (12.7\%). In the subgroup analysis, GGO was the most common imaging finding of the PT $[n=11$ (78.6\%)]. Conversely, the imaging findings of the PT group and the MT group were varied (Figure 3). We compared

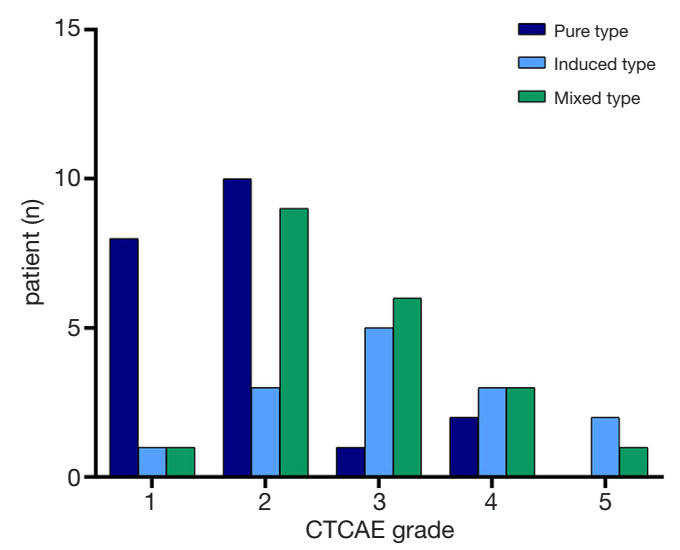

Figure 2 Patients with immune checkpoint inhibitor-related pneumonitis stratified by the Common Terminology Criteria for Adverse Events (CTCAE; version 4:0). the incidence of AIP-ARDS in the 3 groups and found that the incidence in the IT group was higher than those in the PT group and MT group (57.1\%, 9.5\%, and 25.0\%, respectively, $\mathrm{P}=0.010)$. There was an association between AIP-ARDS and severe grade pneumonitis (contingency coefficient $=0.707, \mathrm{P}<0.001$ ).

\section{Management}

Of the 55 participants, $37(67.3 \%)$ were treated with glucocorticoids, including 9 in the PT group, 12 in the IT group, and 16 in the MT group (42.9\%, 85.7\%, and 80.0\%, respectively; $\mathrm{P}=0.014$ ) (Figures 4,5). Participants who had gone without glucocorticoid treatment had grade 1 or 2 CIP. The utilization rates of antibiotics in the IT group and the MT group were significantly higher than those in the PT group $(71.4 \%, 80.0 \%$, and $23.8 \%$, respectively; $\mathrm{P}=0.001$ ) (Figures 4,5). Out of the MT participants, 4 were not treated with antibiotics; these patients did not display complications of infection but did have disease progression $(n=3)$ or radiation-related pneumonitis $(n=1)$. Antiviral treatment was administered in $85.7 \%$ of patients in the IT group, 9.5\% ( $\mathrm{n}=2)$ in the PT group, and $25.0 \%(\mathrm{n}=5)$ in the MT group $(\mathrm{P}<0.001)$ (Figures 4,5). Respiratory support therapy was performed in $4(19.1 \%), 12(85.7 \%)$, and 15 (75.0\%) patients in the PT group, the IT group, and the MT group, respectively (Table 3).

\section{Outcomes}

Except for 3 cases without evaluation outcomes, CIP
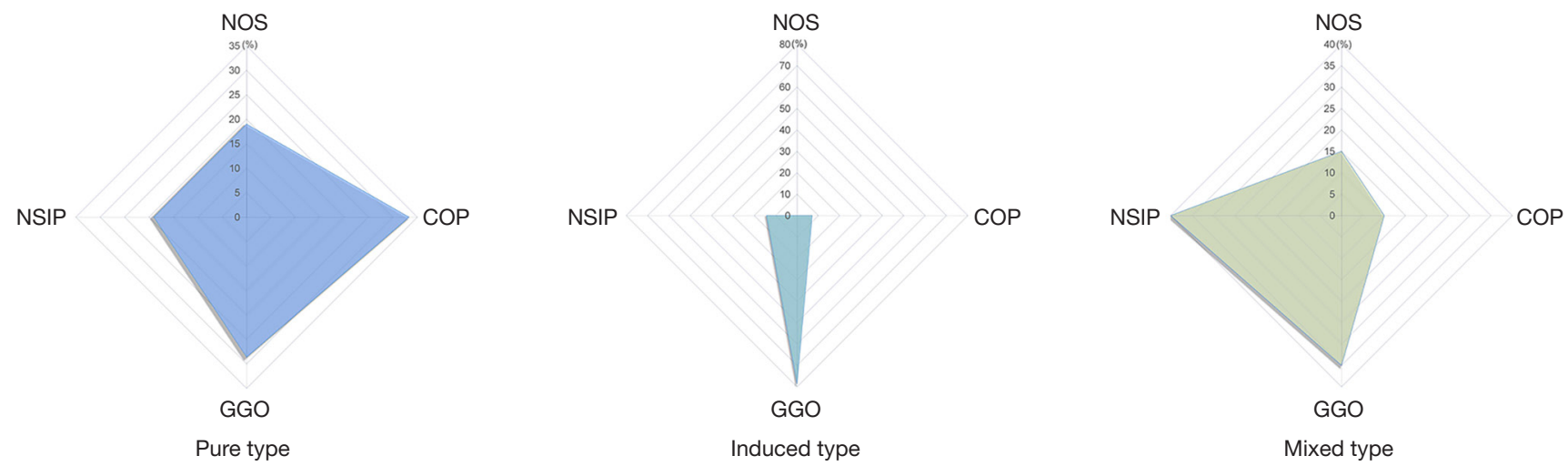

Figure 3 Radar chart of the imaging features of the pure type, induced type, and mixed type of CIP. COP, cryptogenic organizing pneumonitis; GGO, ground glass opacities; NSIP, nonspecific interstitial pneumonia; NOS, pneumonitis not otherwise specified; CIP, immune checkpoint inhibitor-related pneumonitis. 


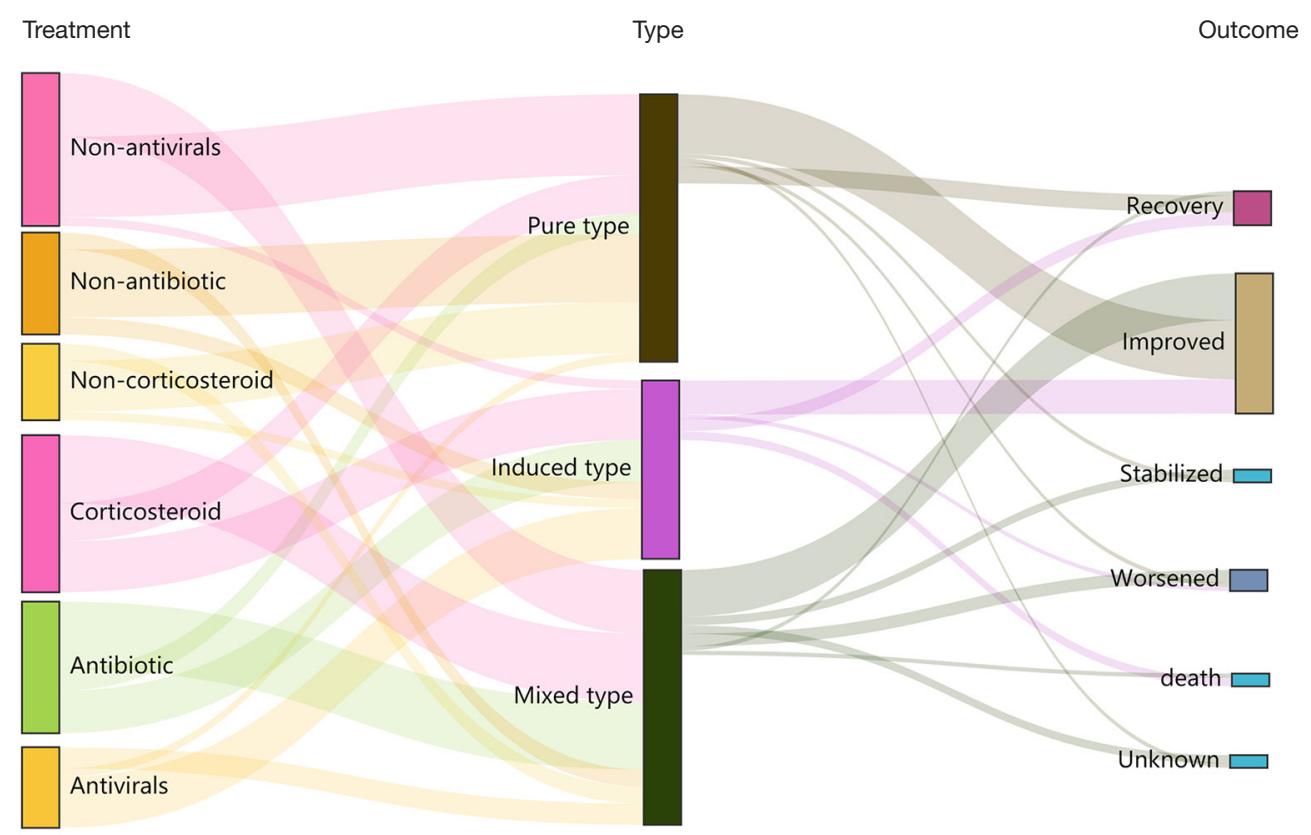

Figure 4 Sankey diagram of the treatments and outcomes with the pure type, induced type, and mixed type CIP. CIP, immune checkpoint inhibitor-related pneumonitis.

resolved in $8(15.4 \%)$ participants, improved in $33(63.4 \%)$, stabilized in $3(5.8 \%)$, and worsened or led to death in 8 (15.4\%) participants (Figures 4,5 ). The improvement rate of participants with the PT was higher than that in those with the IT and MT, but the difference was not significant (90.0\%, 78.6\%, and $66.7 \%$, respectively; $\mathrm{P}=0.223$ ). In the overall population, the median time to improvement was 0.7 (0.2-7.6) months. Furthermore, the median improvement times of the PT, IT, and MT groups were 0.9, 0.5, and 0.3 months, respectively $(\mathrm{P}=0.028)$ (Figure 6). Among the groups, the improvement time after corticosteroid treatment was $0.7(0.1-1.9)$ months in the PT, $0.2(0.1-0.6)$ months in the IT, and $0.2(0.1-0.5)$ months in the MT $(\mathrm{P}=0.048)$.

Logistic regression analysis of the factors associated with the improvement rate of CIP showed that only grades 3-5 were significantly and independently associated with a lower improvement rate [odds ratio $(\mathrm{OR})=0.17,95 \%$ confidence interval $(\mathrm{CI})=0.03-0.92, \mathrm{P}=0.039)($ Table 4$)$.

Except for 8 patients without tumor efficacy evaluation, the objective response rate was $78 \%$ (95\% CI: 52-94) in the PT group, 31\% (95\% CI: 9-61) in the IT group, and 44\% (95\% CI: 20-70) in the MT group ( $\mathrm{P}=0.027$ ) (Table 3). During follow-up, with 22 events of progression and 9 deaths, the median PFS and OS were not reached. A total of 3 deaths were related to CIP, including 2 in the IT group and 1 in the MT group.

\section{Immunotherapy rechallenge}

After recovery/improvement, 21 patients continued immunotherapy, including $13(61.9 \%)$ participants in the PT group, 4 (19.0\%) in the IT group, and $4(19.0 \%)$ in the MT group (Table 3). Among these 21 participants, CIP reoccurred in 2 patients with non-small cell lung cancer (NSCLC), who were both in the MT group. These 2 participants had an initial grade of 2 and developed recurrent grade 4 CIP. They were treated with steroids and immunoglobulin. The participants were still undergoing treatments at the time this manuscript was written.

\section{Discussion}

This real-world, retrospective, observational study indicated that the spectrum of CIP could be newly classified based on clinical factors. Our data described the differences in clinical characteristics, radiological features, treatments, and outcomes among the 3 types.

In recent years, ICIs are widely used for the treatment of lung cancer, whether it is early or advanced lung cancer, as 


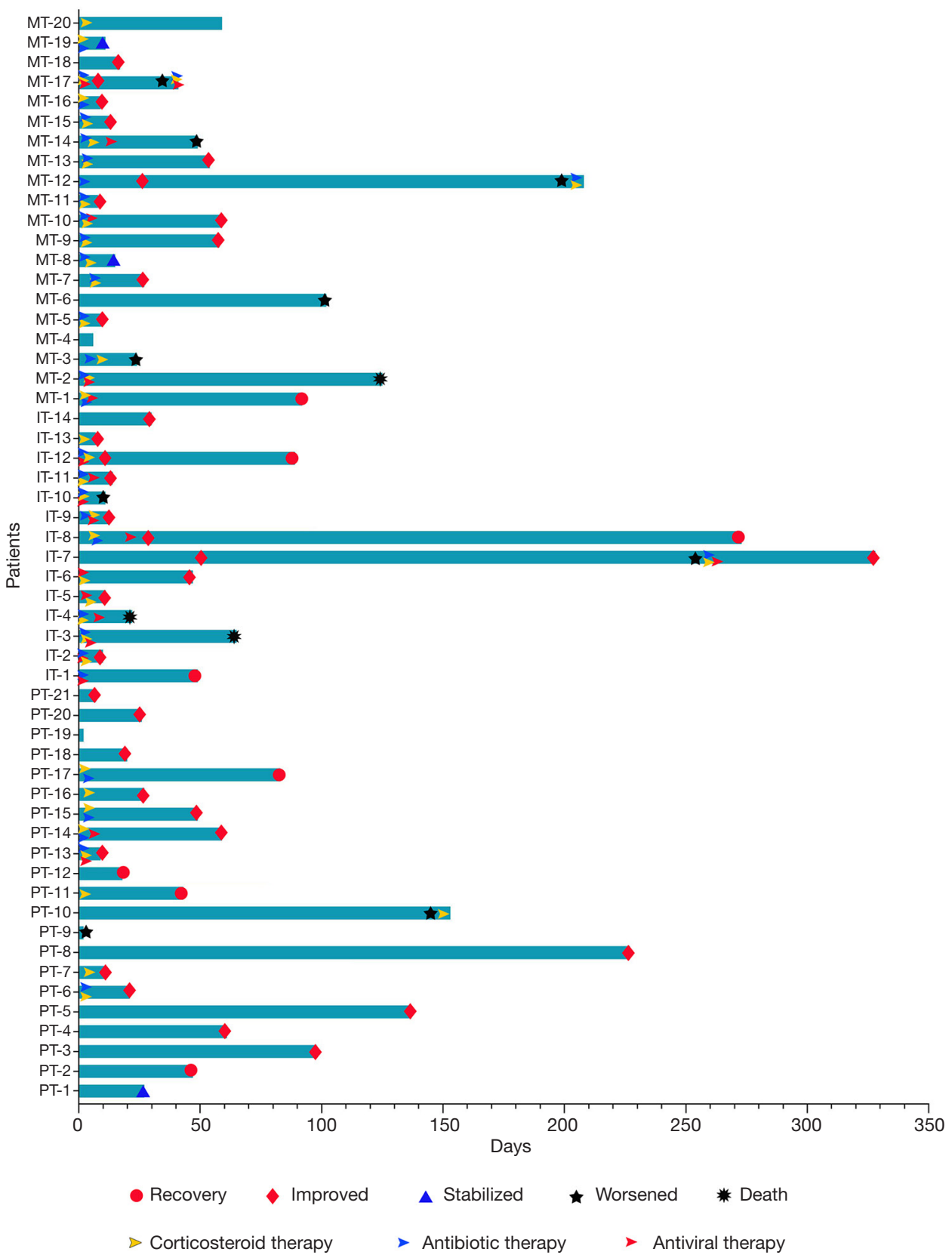

Figure 5 Individual courses of the patients from the onset of pneumonitis to outcomes after treatments. PT, pure type; IT, induced type; MT, mixed type.

well as NSCLC or small cell lung cancer (SCLC). Although studies have demonstrated ICIs can significantly improve clinical outcomes, ICIs may lead to unique irAEs. Grade 1-2 irAEs accounted for the majority, but FAE also occurred. CIP is the most common FAE in PD-1/PD-L1 inhibitors.

In our study, the CIP-related mortality rate was $5.5 \%$, which was lower than those in previous real-world clinical studies $(12.8-22.7 \%)(14,38,39)$. The lower observed mortality rate of CIP may be partially explained by early diagnosis and personalized treatments. The median time from the initiation of immunotherapy to CIP was 2.6 months, which was consistent with previous studies $(34,39)$. 
Table 3 The treatments and outcomes of the new types

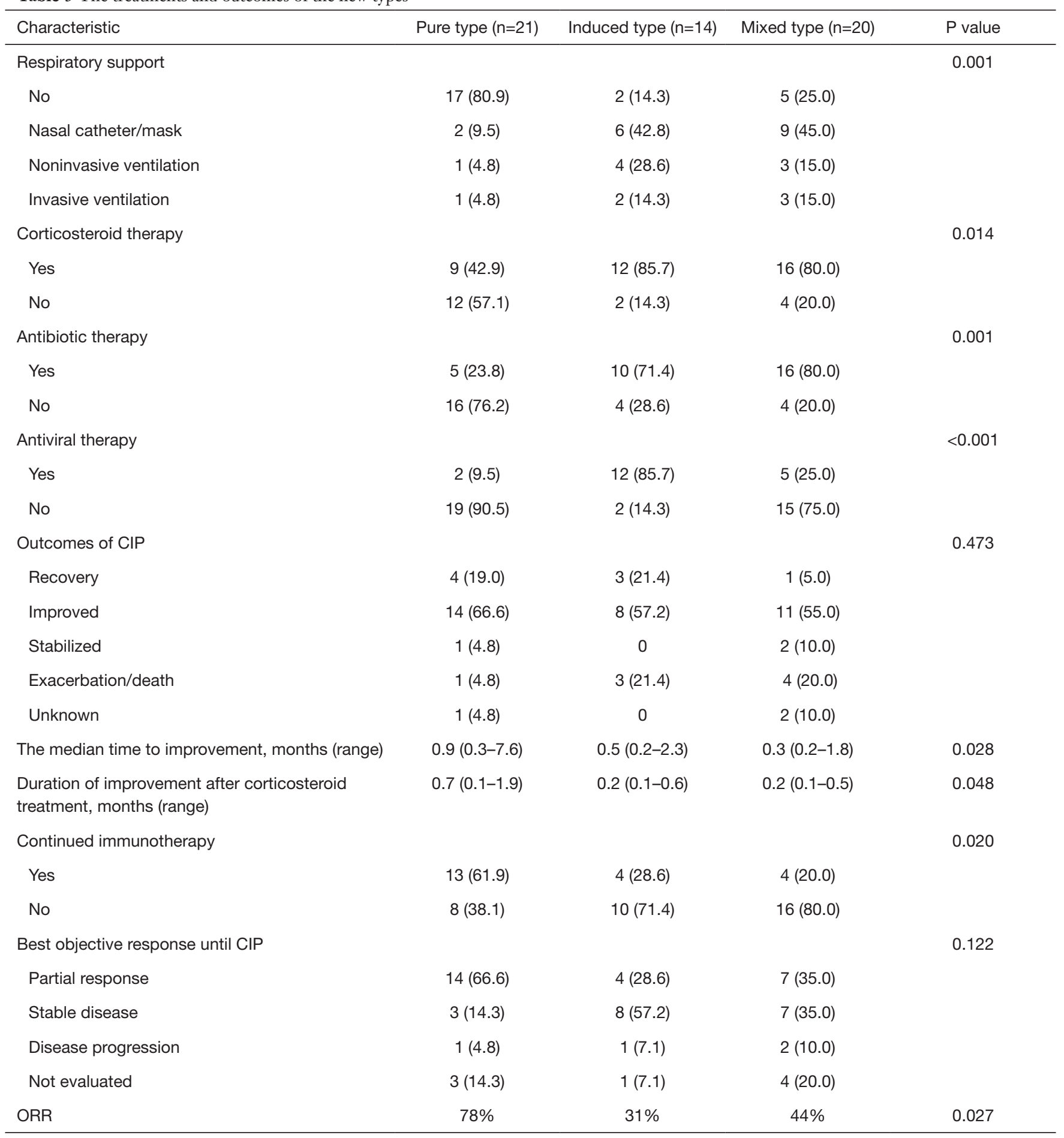

CIP, immune checkpoint inhibitor-related pneumonitis; ORR, object response rate. 
According to clinical factors, the 55 patients were classified as having the PT, IT, or MT of CIP. The IT and MT are both associated with radiotherapy and viral infection, but had different specific associated characteristics. In the IT, phosphoprotein 65 (pp65) and CMV-IgG were positive, but no CMV inclusion bodies were found in pathology, and metagenomics next-generation sequencing (mNGS) of bronchoalveolar lavage fluid (BALF) was negative. Conversely, subjects in the MT developed CIP with cytomegalovirus pneumonia, which was diagnosed by positive CMV culture in BALF or tissue, CMV-DNA in BALF, or CMV inclusion bodies in lung tissue (40).

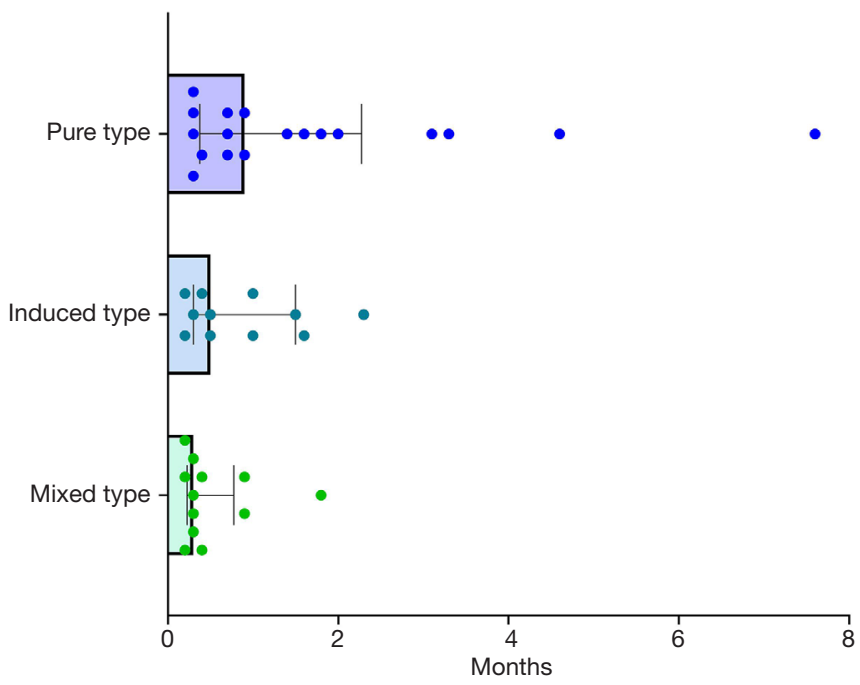

Figure 6 Distribution of the improvement times for the 3 types of pneumonitis. The data are presented as the median (interquartile range).
The IT participants received nonthoracic radiotherapy and subsequently developed CIP. In addition, pneumonitis did not occur in these patients during multiple courses of immunotherapy, but did so after radiotherapy. Patients with MT received thoracic radiotherapy and developed radiation-related pneumonitis combined with CIP.

The proportion of fever, and elevated serum levels of PCT and WBCs in the MT group were higher than those in the other 2 groups. These findings supported the diagnosis of MT. We found that the grade of CIP in the PT group was lower than that in the other 2 groups. Similarly, the PS and mMRC scores in the PT group during pneumonitis was lower $(\mathrm{P}=0.002$ and 0.001 , respectively). Moreover, both of the scores were positively correlated with the grade of pneumonitis. Therefore, the differences in the PS and mMRC scores among the 3 groups may be explained by the grades of CIP. A recent study also reported NSCLC patients with CIP have significantly higher mMRC scores, compared with patients without CIP (41). In our study, $58.2 \%$ of patients had a PS score of $2-4$, and the poor scores were caused by ICIs treatment. These patients met the conditions of severe lung cancer that we proposed in our previous studies (42). Advanced severe lung cancer does not refer to end-stage lung cancer, but refers to various factors inherently associated with the disease or caused by the application of anticancer drugs, a PS score of 2-4, and stage IIIB, IIIC, and IV patients who have the greatest potential to benefit from existing systemic anticancer therapies (42).

In the MT group, $50.0 \%$ of participants developed CIP of grade 3-5. The MT was characterized by a combination of symptoms and imaging changes associated with infection or tumor progression or radiation pneumonitis

Table 4 Factors associated with improvement rate of pneumonitis

\begin{tabular}{|c|c|c|c|c|}
\hline Variable & \multicolumn{2}{|c|}{ Univariate analysis } & \multicolumn{2}{|c|}{ Multivariable analysis } \\
\hline Age $\geq 65$ years & $3.00(0.71-12.74)$ & 0.131 & $1.75(0.30-10.20)$ & 0.533 \\
\hline Male vs. female & $0.69(0.13-3.73)$ & 0.666 & $2.43(0.20-29.69)$ & 0.487 \\
\hline Smoker & $0.58(0.16-2.16)$ & 0.420 & $0.29(0.04-3.21)$ & 0.291 \\
\hline Radiotherapy at baseline & $0.53(0.11-2.54)$ & 0.427 & $0.37(0.04-3.21)$ & 0.367 \\
\hline $\mathrm{ICl}$ treatment $\geq 2^{\text {nd }}$ line & $0.25(0.03-0.99)$ & 0.048 & $0.40(0.08-2.06)$ & 0.272 \\
\hline Histology (squamous vs. nonsquamous) & $1.48(0.38-5.73)$ & 0.572 & $3.17(0.51-19.65)$ & 0.214 \\
\hline
\end{tabular}

$\mathrm{ICl}$, immune checkpoint inhibitor; CIP, immune checkpoint inhibitor-related pneumonitis; OR, odds ratio; Cl, confidence interval. 


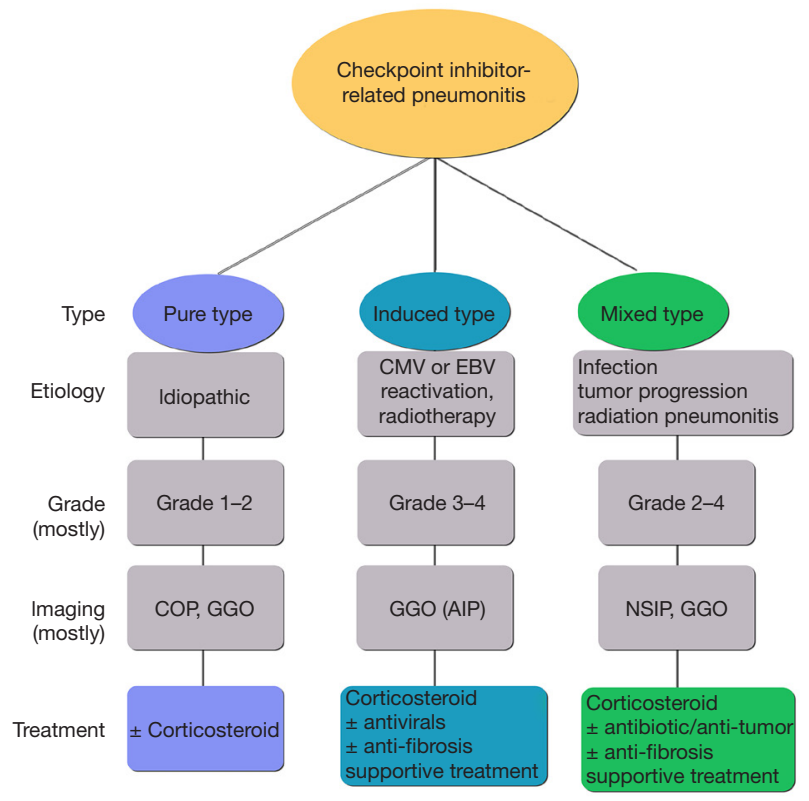

Figure 7 The etiology, grade, radiological features, and management of the 3 types of pneumonitis. CMV, cytomegalovirus; EBV, Epstein-Barr virus; COP, cryptogenic organizing pneumonitis; GGO, ground glass opacities; AIP, acute interstitial pneumonia.

in addition to changes in CIP. Hence, the incidence of severe pneumonitis was higher in the MT group than in the PT group. Severe pneumonitis occurred in $71.4 \%$ of the participants in the IT, and $20 \%$ of these cases were fatal. The mechanism of induced pneumonitis may have been that the activated virus or radiotherapy induced the related antigens to be exposed, which led to a T-cell response to encoded antigens and thus caused CIP (23). The detailed mechanism of the IT leading to severe CIP remains unclear. Radiotherapy induction led to the development of CIP in 4 patients; they did not develop pneumonitis before nonthoracic radiotherapy but rapidly developed pneumonitis after radiation therapy. Pneumonitis occurs in previously irradiated lungs, which is known as radiation recall pneumonitis (RRP) (43-45). Contrastingly, tumor regression in the unirradiated field has been found, which is known as the abscopal effect (46-48). However, no report of CIP after nonthoracic radiotherapy is available. The specific mechanisms of distant radiotherapy leading to CIP remain unclear.

Our study revealed that GGO is a common feature regardless of the type of CIP. Previous studies have also shown GGO as the major imaging finding $(34,49)$.
Among the 3 groups, GGO accounted for the highest proportion $(78.6 \%)$ in the IT. One study indicated that GGO was a significant predictor of worse OS (50). In the current study, AIP-ARDS was associated with severe grade CIP, which was a risk factor for a low improvement rate of CIP. The incidence of AIP-ARDS in the IT group was higher than that in the other 2 groups. Above all, the IT may result in a worse OS; however, OS has not been reached in the current study.

According to the guidelines, the graded treatment of CIP is based on systemic glucocorticoids, supplemented by empirical antibiotics and immunosuppressive agents when necessary. The treatment of CIP in the PT group basically followed the guidelines. In the IT group, in addition to steroid therapy, $85.7 \%$ of patients were given antiviral therapy (ganciclovir antiviral agent). It has been shown that the use of immunosuppressants in checkpoint inhibitorinduced colitis with CMV reactivation can lead to a severe inflammatory response and viral spread, which suggests the importance of diagnosis and treatment of CMV infection (51-53). Two patients in the PT and 3 patients in the MT group were diagnosed with severe pneumonitis and were given antiviral treatment empirically. Nevertheless, no evidence of viral infection or activation was found upon later examination. In the MT group, 2 patients received antiviral treatment due to viral pneumonia, and $80 \%$ of participants with bacterial pneumonia were treated with antibiotics, even if they had grade 1-2 CIP. Glucocorticoid therapy alone in the MT may aggravate or spread the infection. Antibiotic treatment was given to 5 patients in the PT group and 10 patients in the IT group, due to the severity of their CIP or suspision of bacterial pneumonia at the time of early diagnosis. According to the guidelines, antibiotics can be used prophylactically in patients with grade 3-4 pneumonitis. However, no signs of infection were found in subsequent tests. Based on the available data, we propose individual clinical management strategies for the 3 types: for PT patients, glucocorticoid graded treatment is recommended; for IT patients, in addition to corticosteroids and supportive treatment, antiviral therapy (for virusinduced CIP), and anti-fibrotic therapy (for radiotherapyinduced CIP) can be considered; for MT patients, antibiotic treatment (for co-infection), anti-tumor treatment (for co-tumor progression), and anti-fibrotic therapy (for patients complicated with co-radiation pneumonitis) can be considered (Figure 7).

The patients in the PT group were more likely to have a better prognosis in terms of pneumonitis. We found a lower 
improvement rate in grade 3-5 CIP by logistic regression analyses. The better outcomes of the PT may be associated with lower grade pneumonitis. Interestingly, our study showed that the improvement time of the PT was longer than that of the other 2 groups. As mentioned above, for the MT, the symptoms are superimposed on the infection or progression and can be quickly relieved after anti-infection treatment. In contrast, the patients in the PT group had no specific cause, unlike those in the MT and IT groups, which may be the cause for the longer improvement time. Another reason may be that $38.1 \%$ of patients in the PT group had grade 1 pneumonitis without receiving treatment for CIP. Additionally, grade 1 CIP is asymptomatic, resulting in a prognostic assessment based on imaging, which was more frequent in severe grade CIP than in low-grade CIP. Our study showed that early active treatments of MT and IT may promote rapid improvement, but delayed treatment may result in rapid progression.

We found that the response to corticosteroid therapy was slower in the PT group than in the IT and the MT groups. We hypothesized that patients with the PT had numerous activated T-cells and cytokines released (54), leading to a stronger inflammatory response than that in patients with the other 2 types. The improvement times after glucocorticoid treatment in the 3 groups were different, and thus, whether there were differences in the course of glucocorticoid therapy should be further studied.

In our study, the recurrence rate of immunotherapy rechallenge $(9.5 \%)$ was lower than that in previous studies $(55,56)$. Furthermore, the recurrence rate in the MT group was higher than that in the other 2 groups. Thus, the low recurrence rate may be associated with different types of pneumonitis.

We found a higher ORR (78\%) in the PT group, while the ORR was only $31 \%$ in the IT group. Studies have shown that grade 1-2 CIP was significantly associated with increased efficacy, whereas severe grade CIP was not $(14,57)$. The lower ORR of the IT may be partially explained by the more severe grade pneumonitis of that type. We hypothesize that viruses or radiotherapy activate nontumor-specific antigens without a positive effect on antitumor activity in the IT group. Conversely, patients with the PT have common antigens targeting the tumor and the lung, or autoreactive $\mathrm{T}$ cells, autoantibodies, and cytokines produced by the antitumor response acting on inflammatory organs $(23,54)$.

This study has some limitations. First, this was a realworld retrospective study with a small sample size. Second, our results encompass a narrow time window with limited follow-up duration for some participants. A large-scale prospective cohort study should be conducted to further elucidate the 3 different types of CIP.

In conclusion, our study provides new insights into the classification of CIP. The determination of new classifications could favor strategies for the treatment of CIP and the prediction of the tumor response to ICIs.

\section{Acknowledgments}

The authors appreciate the academic support from AME Lung Cancer Collaborative Group.

Funding: This study was supported by Guangdong HighLevel University Clinical Cultivation Project (2017-21020), Guangzhou Science and Technology Program key projects (202008010006), and Wu Jieping Medical Foundation (20201101).

\section{Footnote}

Reporting Checklist: The authors have completed the STROBE reporting checklist. Available at http://dx.doi. org/10.21037/tlcr-20-1258

Data Sharing Statement: Available at http://dx.doi. org/10.21037/tlcr-20-1258

Conflicts of Interest: All authors have completed the ICMJE uniform disclosure form (available at http://dx.doi. org/10.21037/tlcr-20-1258). Gregg JP reports that he is a speaker for AstraZeneca and BMS on non-branded content associated with tissue stewardship. Song Y serves as an unpaid editorial board member of Translational Lung Cancer Research from Sep 2014 to Aug 2020 and Sep 2020 to Aug 2022. The other authors have no conflicts of interest to declare.

Ethical Statement: The authors are accountable for all aspects of the work in ensuring that questions related to the accuracy or integrity of any part of the work are appropriately investigated and resolved. All procedures performed in this study involving human participants were in accordance with the Declaration of Helsinki (as revised in 2013). Institutional review board/ethics committee approval was obtained from the Institutional Review Board of the First Affiliated Hospital of Guangzhou Medical University (Guangzhou, Guangdong, China) (No. 2020-95). Individual 
consent for this retrospective analysis was waived.

Open Access Statement: This is an Open Access article distributed in accordance with the Creative Commons Attribution-NonCommercial-NoDerivs 4.0 International License (CC BY-NC-ND 4.0), which permits the noncommercial replication and distribution of the article with the strict proviso that no changes or edits are made and the original work is properly cited (including links to both the formal publication through the relevant DOI and the license). See: https://creativecommons.org/licenses/by-nc-nd/4.0/.

\section{References}

1. Tan PS, Aguiar PJ, Haaland B, et al. Comparative effectiveness of immune-checkpoint inhibitors for previously treated advanced non-small cell lung cancer A systematic review and network meta-analysis of 3024 participants. Lung Cancer 2018;115:84-8.

2. Jiang T, Zhou C, Hu J, Song Y. Combination immune checkpoint inhibitors with platinum-based chemotherapy in advanced non-small cell lung cancer: what's known and what's next. Transl Lung Cancer Res 2019;8:S447-50.

3. Michot JM, Bigenwald C, Champiat S, et al. Immunerelated adverse events with immune checkpoint blockade: a comprehensive review. Eur J Cancer 2016;54:139-48.

4. Zhao B, Zhao H, Zhao J. Fatal adverse events associated with programmed cell death protein 1 or programmed cell death-ligand 1 monotherapy in cancer. Ther Adv Med Oncol 2020;12:1758835919895753.

5. Chuzi S, Tavora F, Cruz M, et al. Clinical features, diagnostic challenges, and management strategies in checkpoint inhibitor-related pneumonitis. Cancer Manag Res 2017;9:207-13.

6. Rahouma M, Baudo M, Yahia M, et al. Pneumonitis as a complication of immune system targeting drugs?-a meta-analysis of anti-PD/PD-L1 immunotherapy randomized clinical trials. J Thorac Dis 2019;11:521-34.

7. Rashdan S, Minna JD, Gerber DE. Diagnosis and management of pulmonary toxicity associated with cancer immunotherapy. Lancet Respir Med 2018;6:472-8.

8. Cadranel J, Canellas A, Matton L, et al. Pulmonary complications of immune checkpoint inhibitors in patients with nonsmall cell lung cancer. Eur Respir Rev 2019;28:190058.

9. Thompson JA, Schneider BJ, Brahmer J, et al. Management of Immunotherapy-Related Toxicities, Version 1.2019. J Natl Compr Canc Netw2019;17:255-89.
10. Haanen JBAG, Carbonnel F, Robert C, et al. Management of toxicities from immunotherapy: ESMO Clinical Practice Guidelines for diagnosis, treatment and followup. Ann Oncol 2018;29:iv264-6.

11. Brahmer JR, Lacchetti C, Schneider BJ, et al. Management of Immune-Related Adverse Events in Patients Treated With Immune Checkpoint Inhibitor Therapy: American Society of Clinical Oncology Clinical Practice Guideline. J Clin Oncol 2018;36:1714-68.

12. Grangeon M, Tomasini P, Chaleat S, et al. Association Between Immune-related Adverse Events and Efficacy of Immune Checkpoint Inhibitors in Non-small-cell Lung Cancer. Clin Lung Cancer 2019;20:201-7.

13. Cortellini A, Chiari R, Ricciuti B, et al. Correlations Between the Immune-related Adverse Events Spectrum and Efficacy of Anti-PD1 Immunotherapy in NSCLC Patients. Clin Lung Cancer 2019;20:237-47.e1.

14. Tone M, Izumo T, Awano N, et al. High mortality and poor treatment efficacy of immune checkpoint inhibitors in patients with severe grade checkpoint inhibitor pneumonitis in non-small cell lung cancer. Thorac Cancer 2019;10:2006-12.

15. Zhou X, Yao Z, Yang H, et al. Are immune-related adverse events associated with the efficacy of immune checkpoint inhibitors in patients with cancer? A systematic review and meta-analysis. BMC Med 2020;18:87.

16. Fujita K, Kim YH, Kanai O, et al. Emerging concerns of infectious diseases in lung cancer patients receiving immune checkpoint inhibitor therapy. Respir Med 2019;146:66-70.

17. Del Castillo M, Romero FA, Argüello E, et al. The Spectrum of Serious Infections Among Patients Receiving Immune Checkpoint Blockade for the Treatment of Melanoma. Clin Infect Dis 2016;63:1490-3.

18. Su Q, Zhu EC, Wu JB, et al. Risk of Pneumonitis and Pneumonia Associated With Immune Checkpoint Inhibitors for Solid Tumors: A Systematic Review and Meta-Analysis. Front Immunol 2019;10:108.

19. Shaverdian N, Lisberg AE, Bornazyan K, et al. Previous radiotherapy and the clinical activity and toxicity of pembrolizumab in the treatment of non-small-cell lung cancer: a secondary analysis of the KEYNOTE-001 phase 1 trial. Lancet Oncol 2017;18:895-903.

20. Hwang WL, Pike L, Royce TJ, et al. Safety of combining radiotherapy with immune-checkpoint inhibition. Nat Rev Clin Oncol 2018;15:477-94.

21. Lu CS, Liu JH. Pneumonitis in cancer patients receiving anti-PD-1 and radiotherapies: Three case reports. 
Medicine (Baltimore) 2017;96:e5747.

22. Liang X, Guan Y, Zhang B, et al. Severe Immune-Related Pneumonitis With PD-1 Inhibitor After Progression on Previous PD-L1 Inhibitor in Small Cell Lung Cancer: A Case Report and Review of the Literature. Front Oncol 2019;9:1437.

23. Das S, Johnson DB. Immune-related adverse events and anti-tumor efficacy of immune checkpoint inhibitors. J Immunother Cancer 2019;7:306.

24. Johnson DB, Balko JM, Compton ML, et al. Fulminant Myocarditis with Combination Immune Checkpoint Blockade. N Engl J Med 2016;375:1749-55.

25. Berner F, Bomze D, Diem S, et al. Association of Checkpoint Inhibitor-Induced Toxic Effects With Shared Cancer and Tissue Antigens in Non-Small Cell Lung Cancer. JAMA Oncol 2019;5:1043-7.

26. Postow MA, Sidlow R, Hellmann MD. Immune-Related Adverse Events Associated with Immune Checkpoint Blockade. N Engl J Med 2018;378:158-68.

27. Chaput N, Lepage $\mathrm{P}$, Coutzac C, et al. Baseline gut microbiota predicts clinical response and colitis in metastatic melanoma patients treated with ipilimumab. Ann Oncol 2017;28:1368-79.

28. Gopalakrishnan V, Spencer CN, Nezi L, et al. Gut microbiome modulates response to anti-PD-1 immunotherapy in melanoma patients. Science 2018;359:97-103.

29. Lin X, Lu T, Li S, et al. Cytomegalovirus infection as an underestimated trigger for checkpoint inhibitor-related pneumonitis in lung cancer: a retrospective study. Clin Transl Oncol 2020. [Epub ahead of print]. doi: 10.1007/ s12094-020-02432-5.

30. Johnson DB, McDonnell WJ, Gonzalez-Ericsson PI, et al. A case report of clonal EBV-like memory CD4(+) $T$ cell activation in fatal checkpoint inhibitor-induced encephalitis. Nat Med 2019;25:1243-50.

31. Travis WD, Brambilla E, Nicholson AG, et al. The 2015 World Health Organization Classification of Lung Tumors: Impact of Genetic, Clinical and Radiologic Advances Since the 2004 Classification. J Thorac Oncol 2015;10:1243-60.

32. Sears CR, Peikert T, Possick JD, et al. Knowledge Gaps and Research Priorities in Immune Checkpoint Inhibitorrelated Pneumonitis. An Official American Thoracic Society Research Statement. Am J Respir Crit Care Med 2019;200:e31-43.

33. Shannon VR. Pneumonitis associated with immune checkpoint inhibitors among patients with non-small cell lung cancer. Curr Opin Pulm Med 2020;26:326-40.

34. Naidoo J, Wang X, Woo KM, et al. Pneumonitis in Patients Treated With Anti-Programmed Death-1/ Programmed Death Ligand 1 Therapy. J Clin Oncol 2017;35:709-17.

35. American Thoracic Society; European Respiratory Society. American Thoracic Society/European Respiratory Society International Multidisciplinary Consensus Classification of the Idiopathic Interstitial Pneumonias. This joint statement of the American Thoracic Society (ATS), and the European Respiratory Society (ERS) was adopted by the ATS board of directors, June 2001 and by the ERS Executive Committee, June 2001. Am J Respir Crit Care Med 2002;165:277-304.

36. Travis WD, Costabel U, Hansell DM, et al. An official American Thoracic Society/European Respiratory Society statement: Update of the international multidisciplinary classification of the idiopathic interstitial pneumonias. Am J Respir Crit Care Med 2013;188:733-48.

37. Eisenhauer EA, Therasse P, Bogaerts J, et al. New response evaluation criteria in solid tumours: revised RECIST guideline (version 1.1). Eur J Cancer 2009;45:228-47.

38. Cho JY, Kim J, Lee JS, et al. Characteristics, incidence, and risk factors of immune checkpoint inhibitor-related pneumonitis in patients with non-small cell lung cancer. Lung Cancer 2018;125:150-6.

39. Suresh K, Voong KR, Shankar B, et al. Pneumonitis in Non-Small Cell Lung Cancer Patients Receiving Immune Checkpoint Immunotherapy: Incidence and Risk Factors. J Thorac Oncol 2018;13:1930-9.

40. Travi G, Pergam SA. Cytomegalovirus pneumonia in hematopoietic stem cell recipients. J Intensive Care Med 2014:29:200-12.

41. Suzuki Y, Karayama M, Uto T, et al. Assessment of Immune-Related Interstitial Lung Disease in Patients With NSCLC Treated with Immune Checkpoint Inhibitors: A Multicenter Prospective Study. J Thorac Oncol 2020;15:1317-27.

42. Xie Z, Zhou C, Qin Y, et al. Diagnosis and treatment strategy for advanced severe lung cancer. Chinese Journal of Practical Internal Medicine 2019;39:416-9.

43. Shibaki R, Akamatsu H, Fujimoto M, et al. Nivolumab induced radiation recall pneumonitis after two years of radiotherapy. Ann Oncol 2017;28:1404-5.

44. Nakamura K, Okubo K, Takahashi T, et al. Radiation recall pneumonitis induced by nivolumab in a patient with renal cell carcinoma. IJU Case Rep 2018;2:30-3.

45. Kalisz KR, Ramaiya NH, Laukamp KR, Gupta A. 
Immune Checkpoint Inhibitor Therapy-related

Pneumonitis: Patterns and Management. Radiographics 2019;39:1923-37.

46. Brix N, Tiefenthaller A, Anders H, et al. Abscopal, immunological effects of radiotherapy: Narrowing the gap between clinical and preclinical experiences. Immunol Rev 2017;280:249-79.

47. Dagoglu N, Karaman S, Caglar HB, et al. Abscopal Effect of Radiotherapy in the Immunotherapy Era: Systematic Review of Reported Cases. Cureus 2019;11:e4103.

48. Mondini M, Levy A, Meziani L, et al. Radiotherapyimmunotherapy combinations - perspectives and challenges. Mol Oncol 2020;14:1529-37.

49. Nobashi TW, Nishimoto Y, Kawata Y, et al. Clinical and radiological features of immune checkpoint inhibitorrelated pneumonitis in lung cancer and non-lung cancers. Br J Radiol 2020;93:20200409.

50. Watanabe S, Ota T, Hayashi M, et al. Prognostic significance of the radiologic features of pneumonitis induced by anti-PD-1 therapy. Cancer Med 2020;9:3070-7.

51. Uslu U, Agaimy A, Hundorfean G, et al. Autoimmune Colitis and Subsequent CMV-induced Hepatitis

Cite this article as: Lin $\mathrm{X}$, Deng $\mathrm{H}$, Chen $\mathrm{L}, \mathrm{Wu} \mathrm{D}$, Chen $\mathrm{X}$, Yang Y, Chen T, Xie X, Xie Z, Liu M, Ouyang M, Qin Y, Li S, Zhong N, Gregg JP, Horita N, Song Y, Zhou C. Clinical types of checkpoint inhibitor-related pneumonitis in lung cancer patients: a multicenter experience. Transl Lung Cancer Res 2021;10(1):415-429. doi: 10.21037/tlcr-20-1258
After Treatment With Ipilimumab. J Immunother 2015;38:212-5.

52. Franklin C, Rooms I, Fiedler M, et al. Cytomegalovirus reactivation in patients with refractory checkpoint inhibitor-induced colitis. Eur J Cancer 2017;86:248-56.

53. Lankes K, Hundorfean G, Harrer T, et al. Anti-TNFrefractory colitis after checkpoint inhibitor therapy: Possible role of CMV-mediated immunopathogenesis. Oncoimmunology 2016;5:e1128611.

54. Esfahani K, Elkrief A, Calabrese C, et al. Moving towards personalized treatments of immune-related adverse events. Nat Rev Clin Oncol 2020;17:504-15.

55. Delaunay M, Cadranel J, Lusque A, et al. Immunecheckpoint inhibitors associated with interstitial lung disease in cancer patients. Eur Respir J 2017;50:1700050.

56. Nishino M, Ramaiya NH, Awad MM, et al. PD-1 Inhibitor-Related Pneumonitis in Advanced Cancer Patients: Radiographic Patterns and Clinical Course. Clin Cancer Res 2016;22:6051-60.

57. Cui P, Huang D, Wu Z, et al. Association of immunerelated pneumonitis with the efficacy of PD-1/PD-L1 inhibitors in non-small cell lung cancer. Ther Adv Med Oncol 2020;12:1758835920922033. 